\title{
CORRELATION BETWEEN PLASMA CORTISOL AND REACTIVITY IN FIVE HORSES
}

\author{
Alexandra Nicoleta PĂVĂLOIU*, PhD Student \\ Professor lonel PAPUC*, PhD
}

\begin{abstract}
A plasma cortisol measurement and behavioral assessment of five horses was performed, to determine whether there was a correlation between plasma cortisol and their reactivity. A subjective emotionality survey for each horse was completed by the horse's owners and an objective novel stimulus reactivity test was performed. The horses reactions were used to calculate reactivity scores on a reactivity assessment sheet we specially designed. Concentration of plasma cortisol was also measured and reactions to the blood sampling were quantified. Associations were made between those different parameters. All five horses appeared to be hypo reactive and their cortisol levels were also very low. Behavioral assessed reactivity seemed to correlate directly with the plasma cortisol, all values showing a marked hypo reactivity in all horses. The data obtained provided evidence that low plasma cortisol is a good marker of reactivity in hypo reactive horses and also that the designed sheets for the assessment of objective and subjective reactivity may be used, in correlation with plasma cortisol as an assessment tool in the current practice.
\end{abstract}

Keywords: horse, reactivity, behavioral tests, plasma cortisol, assessment sheet

\section{Introduction}

Horses are kept for various reasons, having become very popular for sport and recreations in the past decades. Even though when buying a horse, appearance and performance is being assessed, temperament is not. Therefore, many horses are being sold due to their undesirable temperament and a match between horse and its owners temperaments seems now a logical and necessary factor (Visser et al., 2003). Romania is now approaching this field of interest in the past years, so an assessment method adapted to its economic and social reality seems appropriate.

There are many reasons why horse behavior is important even to the clinician. This applies even more in equine medicine then in small animal medicine, because the size of the patient represents more of a threat. The greatest proportion of horse related injury and death is rather behavioral then performance related. Therefore, the first reason to be considered is owner safety, and also self protection as a veterinarian (Houpt et al., 2006).

Temperament in animals has only been of interest in the last half of century, with Pavlov as a pioneer and followed by a few scientist in the 70's, followed by more attention from researchers in

\footnotetext{
* University of Agricultural Sciences and Veterinary Medicine Cluj Napoca, Faculty of Veterinary Medicine, Discipline of Semiology, Ethology and Diagnostic Imaging, 3-5 Mănăştur Street, 400372 Cluj-Napoca, Romania, e-mail: alexa.nicoleta@gmail.com 
the late 90's. It can be defined as an association of internal factors that assure a constant behavior from one moment to another, behavior that is constant in time and correlated to other behavior (Lansade, 2005).

Reactivity or emotionality, in the horse, is a heightened state of arousal, which may influence the horse's manageability and usefulness for specific tasks. Reactivity in horses has both behavioral (emotionality, flight response etc.) and physiological (changes in heart rate, respiration, hormones like adrenaline and cortisol etc.) components, and these components should be used as an assessment tool by correlation (McCall et al., 2006).

Potential application of horse personality and temperament assessment have been researched in Lansade (2005). Equine personality assessment currently involves classification and assessment of observed behavior (Anderson et al., 1999, Visser et al., 2002, McCall et al., 2006). Also, completion of subjective questionnaires on the behavior of individuals over a variety of situations was performed (Momozawa et al., 2005, Visser et al., 2003) as well as comparative research (Gosling et al., 2002). There were also studies performed on whether judges can actually agree on the assessment of horse personality characteristics (Morris et al., 2002). Reactivity was measured either by novel stimulus tests or isolation tests (Lansade et al., 2008, McCall et al., 2006).

This study was conducted to identify whether there is a correlation between plasma cortisol and horse reactivity, and also to design two assessment sheets, one for an objective assessment and one for the owners, as a subjective assessment. Should significant relationships exist, they may prove useful in predicting the suitability of horses for different equestrian sports.

\section{Material and Method}

\section{The horses}

A total of 5 horses were used, aged between 2 and 20 years, of different breeds ( 3 mares of half bred Romanian Heavy Draught, one Nonius half bred stallion and one Romanian Sport Horse stallion). Two of them were from the Faculty of Veterinary Medicine Cluj Napoca and three belonged to different private owners in Cluj Napoca.

\section{Blood collection}

Because prior studies have shown circadian rhythms in cortisol concentrations (Frank, 2006, Higgins et al., 2006), blood sampling began at $10 \mathrm{AM}$ and ended at $11 \mathrm{AM}$ each collection day. To avoid stress, collection was performed in the horse's natural environment, whether it was the stable or a paddock, and the horse was held by a person that was familiar to him. Blood collection was done by venipuncture of the left jugular vein and one 2,0 ml blood sample was collected into a sterile vacutainer containing $0,1 \mathrm{ml}$ of $15 \%$ EDTA solution. The behavioral reaction of each horse was scored, by giving a score of ' 0 ' for no reaction, a score of ' 1 ' for minimal movement and a score of '2' was given for extended movement. Blood samples were kept on ice immediately after collection and centrifuged for plasma within an hour after collection.

\section{Plasma cortisol determination}

Plasma cortisol was assessed by ELFA (enzyme linked fluorescent immunoassay) technique. Its principle relies on the combination of a traditional ELISA (enzyme linked immunosorbent assay) test with a final fluorescent detection. A CORS test was used on a Vitek ImmunoDiagnostic Assay System. CORS VIDAS Method is calibrated by the reference method ID-GCMS (Isotope Dilution- Gas Cromatography Mass Spectrometry). 
Plasma cortisol levels were determined at a local human accredited laboratory, since no special equine kits are commercially available in Romania for the moment.

\section{Subjective scoring}

Each horse was assigned with a subjective emotionality score adapted from McCall et al., 2006, by their owners. The scores were included in a specially designed subjective assessment sheet that contained data on the assessed horse (Name, Breed, Age, Sex) and description of a possible scenario. Emotionality scores that were to be assigned were as following: 1- bold and calm, 2- bold, 3-normal, 4-shy, 5-very shy and reactive (McCall et al., 2006).

\section{Objective reactivity testing}

The next day after blood collection was used for objective reactivity testing by novel stimuli test, as adapted from Anderson et al (1999). The first stimulus used was a vocalizing, dancing toy frog placed in front of the horse for 20 seconds. After the cease of any reaction to this stimulus, a 'popping balloon' test (Fig. 1) was used and then an umbrella was opened in front of the horse (Fig. 2).

The horses were allowed to touch and inspect the stimuli after confronted with them. The tests were performed in an open space, with which the horses were familiar with and they were handled by the same handler. Behavior was observed and noted on a assessment sheet we designed,

that contained data on the horses (name, age, sex, breed), scores from the reactions to the blood sampling, detailed description of expressions, vocalizations and movement, that were based on a behavioral defined scale as described by Anderson et al. (1999), as well as different observations on the clinical appearance of the horses. An average reactivity score was calculated for every horse. The minimum value of the score to the objective testing, after adding the scores from blood collection was 3, while maximum that could be

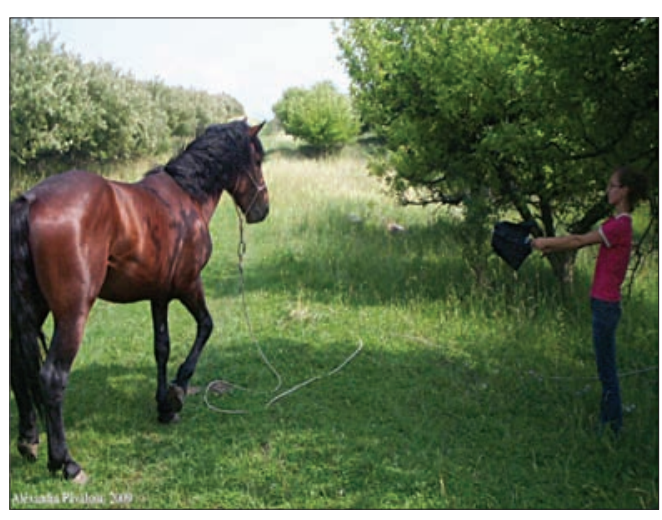

Fig. 1. Preparation for the umbrella test

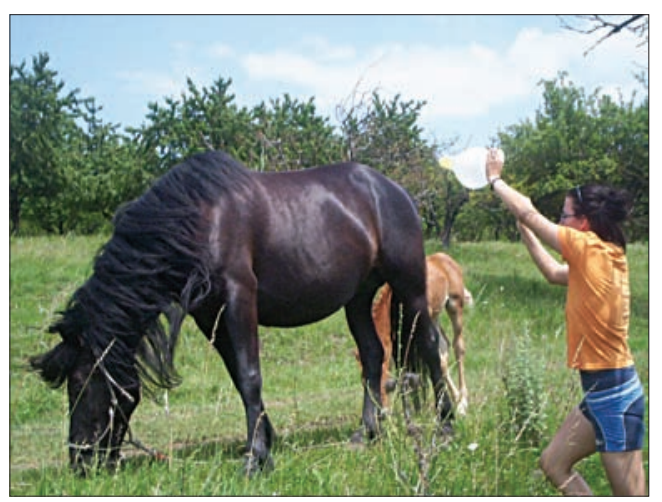

Fig. 2. Popping balloon test obtained was 17, with an average value between 9 and 11 .

\section{Association between different parameters measured}

Association between objectively assessed reactivity and plasma cortisol

Due to the purpose of discovering a relationship between these two parameters, a comparison between each horse's reactivity score and plasma cortisol value was made.

Association between behavioral extremes and plasma cortisol

We compared results of horses with the lowest reactivity score to their plasma cortisol value.

Association between objective and subjective reactivity assessment

To analyze whether owners can rank their horses accurately, we compared results from both the objective behavioral tests and the subjective evaluation sheets of each horse (adapted from Anderson et al. 2006). 


\section{Results}

\section{General}

All horses were easy to work with, none had big issues with blood collection. Reactions to stimuli were diverse, but as an average all horses showed a marked response to the umbrella test, while being practically insensitive to the toy stimulus.

\section{Association between objectively assessed reactivity and plasma cortisol}

Each horse's reactivity score was compared to its plasma cortisol value. As shown in Table 1, 2 and 3, plasma cortisol levels were low in all horses and could be correlated to their corresponding, low objective test scores.

Table 1. Results in plasma cortisol determination, objective and subjective scores

\begin{tabular}{|c|c|c|c|}
\hline Case/sex & Cortisol (ng/ml) & Objective score & Subjective score \\
\hline 1-F & 28,62 & 6 & 2 \\
\hline $2-\mathrm{F}$ & 34,45 & 8 & 1 \\
\hline $3-\mathrm{F}$ & 30,15 & 8 & 2 \\
\hline $4-\mathrm{M}$ & 22,35 & 6 & 2 \\
\hline $5-\mathrm{F}$ & 32,78 & 8 & 1 \\
\hline
\end{tabular}

Table 2. Comparison of obtained basal plasma cortisol value to normal mean value $(55 \mathrm{ng} / \mathrm{ml})$

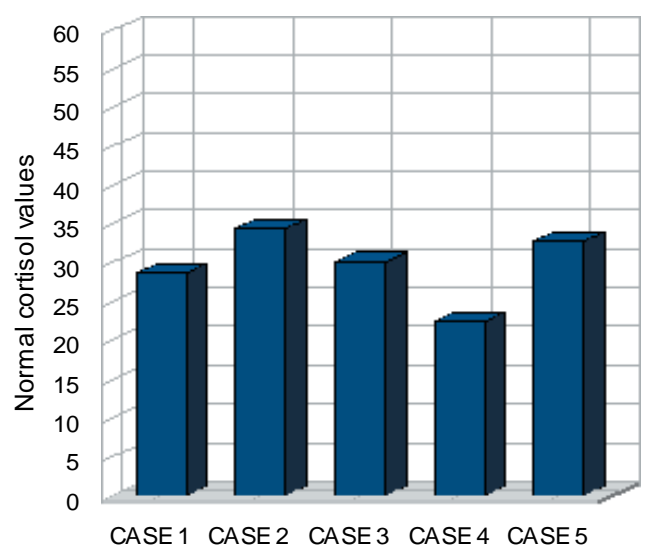

Table 3. Obtained objective scores (average score 9-11)

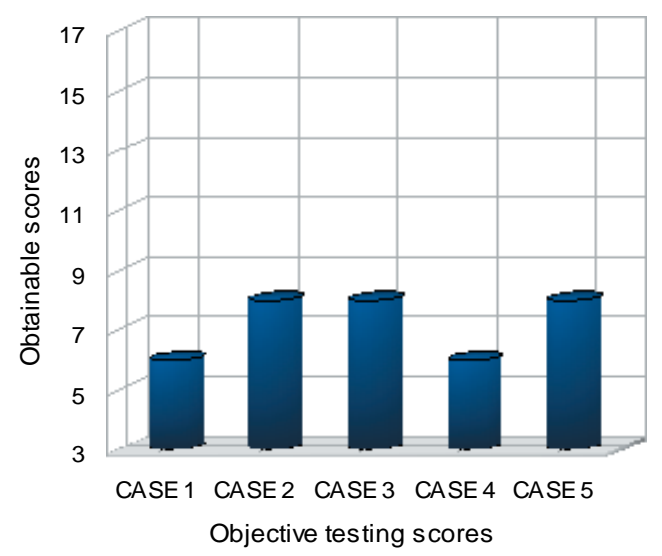

The surprise of the study consisted in the fact that both stallions had very low plasma cortisol levels, as opposed to common belief that they are more aggressive and reactive then mares or geldings.

\section{Association between behavioral extremes and plasma cortisol}

Both extremed of score 6 when assessed by objective testing had the lowest two plasma cortisol values $(22,35$ and $28,62 \mathrm{ng} / \mathrm{ml})$.

\section{Association between objective and subjective reactivity assessment}

All owners assessed their horses as under normal reactivity (hypo reactive, scores 1 and 2), therefore the results correlated well with our measurements.

\section{Discussions}

Basal cortisol levels ranged from 22,35 to $34,45 \mathrm{ng} / \mathrm{ml}$. The values in our cases are in the normal range of plasma cortisol values, but towards the lower limit. It is not to be forgotten that results may vary due to age, sex and breed, as well as other factors (Higgins et al., 2006). 
The surprise of this study was represented by the two stallions with low plasma cortisol levels. Although their hypo reactivity was confirmed by the owner and us in this case (emotionality scores 2 , objective scores 6), stallion reactivity is usually higher than the one of geldings and mares, which is why they are considered not to be suitable as 'pets', and they should rather be used in other purposes than a recreational one.

\section{Conclusions}

A correlation between hormone concentrations, objective scores and subjective scores was found, therefore allowing us to conclude the following: the owners of the five horses tested accurately assessed their horses and low plasma cortisol levels do correlate to low objective test scoring in hypo reactive tested horses. Due to the small number of tested horses and the fact that we only detected hypo reactive horses, we cannot be sure if our results apply to a larger, more reactive group of horses. Our results partially match the studies of Anderson et al. (1996), whose hormone concentration just partly matched objective measurements results and McCall et al. (2006), but a study on a larger group of horses needs to be performed to be sure of the accuracy of this method. In spite of this, we do recommend it as a quick and easy way to assess horse reactivity, since in our case it reflected the horses reactivity status accurately.

\section{Acknowledgments}

The warmest of thanks are addressed to Dr. C. Mureşan, who's patient advice on conceiving an article were of invaluable help. Also, many thanks go to Dr. A. Pîrvulescu, Dr. R. Drozan and Dr. J. Ginter for their assistance on testing.

\section{References}

1. Marsha K. Anderson, Friend, T.H., Evans, J.W., Diana M. Bushong, 1999, Behavioral assessment of horses in therapeutic riding programs, Applied Animal Behaviour Science, 63, 11-24;

2. Frank, N., 2006, Insulin resistance in horses, Proceedings of the American Association of Equine Practitioners;

3. Gosling S.D., Vazire, S., 2002, Are we barking up the right tree? Evaluating a comparative approach to personality, Journal of Res. Personality, 36;

4. Higgins, A. J., Snyder, J. R., 2006, The Equine Manual, Ed. Elsevier Saunders, Philadelphia;

5. Houpt, K.A., Mills, D.S., 2007, Why horse behavior is important to the clinician, Equine Veterinary Journal, 38, 386-387;

6. Lea Lansade, 2005, Le temperament du cheval: Etude theoretique. Application a la selection des chevaux destines a l'equitation, Universite Francois Rabelais de Tours, Ecole Doctorale 'Sante, Sciences et Techniques'.

7. Lea Lansade, Marie-France Boissou, 2008, Reactivity to humans: A temperament trait of horses which is stable across time and situations, Applied Animal Behaviour Science, doi: 10.1016/ j.applanim.2008.04.12;

8. Lea Lansade, Marie-France Boissou, Erhard, H.W., 2008, Reactivity to isolation and association with conspecifics: A temperament trait stable across time and situation, Applied Animal Behaviour Science 109, 355-373;

9. McCall, C.A., Hall, S., McElhenney, W.H., Cummins, K.A., 2006, Evaluation and comparison of four methods of ranking horses based on reactivity, Applied Animal Behaviour Science 96, 15-127;

10. Momozawa, Y, Kusunose R, Kikusui T, Takeuchi Y, Mori Y, 2005, Assessment of equine temperament questionnaire by comparing factor structure between two separate surveys, Applied Animal Behavioural Science, 92, 77-84;

11.Morris, P.H., Gale A., Katherine Duffy, 2002, Can judges agree on the personality of horses?, Personality and Individual Differences 33, 67-81; 
12.Visser, E.K., Van Reenen C.G., Rundgren, M., Zetterqvist, M., Morgan, K., Blokhuis, H.J., 2003, Responses of horses in behavioural tests correlate with temperament assessed by riders, Equine Veterinary Journal, 35.

13.Visser, E.K., Van Reenen, C.G., Van der Werf, J.T.N., Schilder, M.B.H., Knaap, J.H., Barneveld, A., Blokhuis, H.J., 2002, Heart rate and heart rate variability during a novel object test and a handling test in young horses, Psychology and Behavior 76, 289-296. 